\title{
Insights into the Effects of the Dental Stem Cell Secretome on Nerve Regeneration: Towards Cell-Free Treatment
}

\author{
Rohaina Che Man, ${ }^{1}$ Nadiah Sulaiman, ${ }^{1}$ Ruszymah Bt Hj Idrus $\left(\mathbb{D},{ }^{2}\right.$ \\ Shahrul Hisham Zainal Ariffin ${ }^{(D)}{ }^{3,4}$ Rohaya Megat Abdul Wahab, ${ }^{5}$ \\ and Muhammad Dain Yazid $\mathbb{D D}^{1}$ \\ ${ }^{1}$ Tissue Engineering Centre, Universiti Kebangsaan Malaysia Medical Centre, Jalan Yaacob Latif, 56000 Cheras, \\ Kuala Lumpur, Malaysia \\ ${ }^{2}$ Department of Physiology, Faculty of Medicine, Universiti Kebangsaan Malaysia Medical Centre, Jalan Yaacob Latif, 56000 Cheras, \\ Kuala Lumpur, Malaysia \\ ${ }^{3}$ Malaysia Genome Institute (MGI), National Institute of Biotechnology Malaysia (NIBM), Jalan Bangi, 43000 Bangi, \\ Selangor, Malaysia \\ ${ }^{4}$ Faculty of Science and Technology, Universiti Kebangsaan Malaysia, Bangi, Selangor 43600, Malaysia \\ ${ }^{5}$ Department of Orthodontic, Faculty of Dentistry, Universiti Kebangsaan Malaysia, Jalan Raja Muda Abdul Aziz, \\ 50300 Kuala Lumpur, Malaysia
}

Correspondence should be addressed to Muhammad Dain Yazid; dain@ukm.edu.my

Received 5 July 2019; Revised 28 August 2019; Accepted 16 September 2019; Published 24 October 2019

Guest Editor: Drenka Trivanović

Copyright (c) 2019 Rohaina Che Man et al. This is an open access article distributed under the Creative Commons Attribution License, which permits unrestricted use, distribution, and reproduction in any medium, provided the original work is properly cited.

Cell-free treatment is emerging as an alternative to cell delivery to promote endogenous regeneration using cell-derived factors. The purpose of this article was to systematically review studies of the effects of the dental stem cell secretome on nerve regeneration. PubMed and Scopus databases were used where searched and related studies were selected. The primary search identified 36 articles with the utilized keywords; however, only 13 articles met the defined inclusion criteria. Eight out of thirteen articles included in vivo and in vitro studies. We classified the dental stem cell-derived secretome with its nerve regeneration potential. All studies demonstrated that dental stem cell-derived factors promote neurotrophic effects that can mechanistically stimulate nerve regeneration in neurodegenerative diseases and nerve injury. This data collection will enable researchers to gather information to create a precise formulation for future prescribed treatments.

\section{Introduction}

1.1. Neurodegenerative Diseases, Risk Factors, and Current Pharmacotherapy. Neurons are the cells composing the nervous system, including the spinal cord and brain. Neurodegenerative diseases can result when damaged neurons cannot be replaced or reproduced by the body $[1,2]$. This condition is normally related to neuronal structure damage and function failures, factors that cause neuronal death [3]. The significant process of neurodegeneration results in myriad neurodegenerative diseases, including Parkinson's disease (PD), Alzheimer's disease (AD), Huntington's disease
(HD), dementia, and spinal muscular atrophy $[4,5]$. Unfortunately, continuous nerve deterioration, which predominantly affects human brain and spinal cord, is incurable and contributes to movement and mental function problems [6].

The significantly high incidence of neurodegenerative diseases has attracted increased attention in the past decades. PD commonly affects the central nervous system (CNS) and causes abnormal movement that is characterized by progressive loss of muscle control [7]. The projected prevalence of $\mathrm{PD}$ in the US will increase substantially. It is more frequent in men compared to women, with an estimated prevalence of 572 individuals per 100,000 among those aged $\geq 45$ years. 


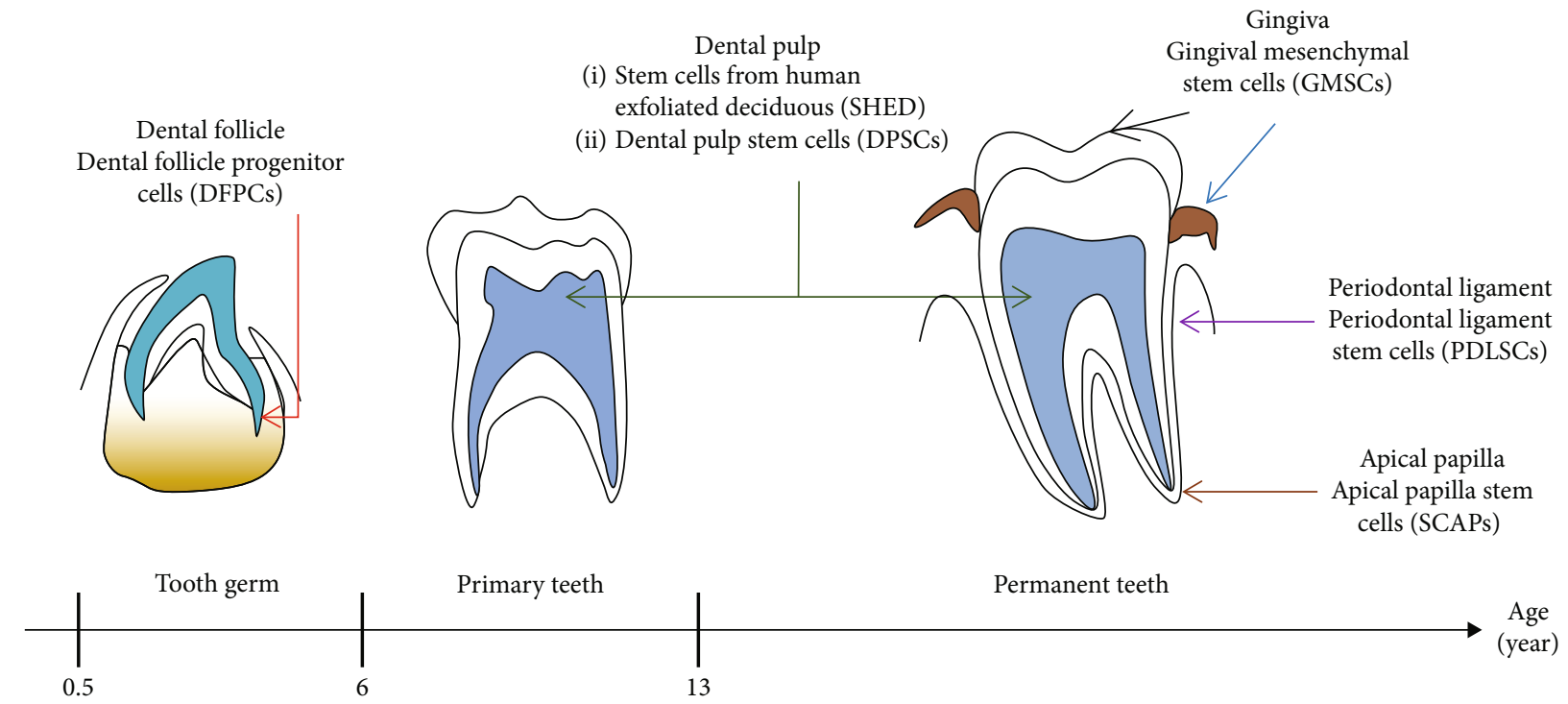

FIgURE 1: Tooth developmental stages with the anatomical localization of the difference dental-derived stem cells in a tooth germ, primary teeth, and permanent teeth. Different subpopulations of DSCs can be categorized according to their tissue of origin. Modified from [74].

These numbers are estimated to increase from 930,000 to $1,238,000$ in 10 years, as projected by the US Census Bureau [8], and this elevation represents a considerable medical problem and social burden. $\mathrm{AD}$ is one of the most common diseases that leads to dementia and depreciation of cognitive function. Approximately 1 million new AD cases are expected to develop every year, with estimated prevalence ranging from 11-16 million [9]. HD is characterized by abnormal cognitive, emotional, and behavioral functions [10]. Intriguingly, the estimated HD prevalence varies up to tenfold depending on the world region. The prevalence in Australia, North America, and Western Europe had escalated over the past 50 years, whereas lower HD rates are reported for Asian populations [11].

Although the etiology for neurodegenerative diseases remains elusive, many recent studies suggest prominent risk factors. Most of the known risk factors include environmental pollutants [12], ageing [13], oxidative stress [14], chemical exposure [15], and infection [16]. There are myriad pharmacotherapies that were investigated to treat the diseases. Acetylcholinesterase inhibitors and N-methyl-d-aspartate (NMDA) receptor agonist both offer a good therapy choice, especially for $\mathrm{AD}$ [17]. In the clinical setting, this particular therapy has attracted significant research interest in order to evaluate the efficacy of pharmacotherapy for $\mathrm{AD}$. A recent study by Manenti et al. [18] revealed significant improvements in motor abilities and a reduction of depressive symptoms in PD patients through anodal transcranial direct current stimulation applied over the dorsolateral prefrontal cortex combined with physical therapy.

In recent decades, researchers have made numerous efforts to elucidate the mechanism(s) of neurodegenerative diseases and possible pharmacotherapies that can help to decelerate and prevent these diseases from worsening. The current medical treatment tends to be palliative rather than curative. Unfortunately, none of them significantly halts the underlying pathology. This review article will expound upon the core value of dental stem cells (DSCs), with special emphasis on dental pulp stem cells (DPSCs) and stem cells from human exfoliated deciduous teeth (SHEDs), and the role of their paracrine factors for potential future applications in neurodegenerative disease therapies.

1.2. DSC Secretome. DSCs can be isolated from various dental soft tissue. They can be divided into several categories according to the origin [19]. Figure 1 shows the anatomical localization of the different DSCs starting from tooth germ, primary teeth, and permanent teeth. Dental follicle progenitor cells (DFPCs) can be isolated from dental follicle tissue of the tooth germ as early as 6 months old. SHEDs can be isolated from primary teeth at 6 years old. Various DSC populations can be isolated from permanent teeth, including DPSCs, periodontal ligament stem cells (PDLSCs), apical papilla stem cells (SCAPs), and gingival mesenchymal stem cells (GMSCs), which can be isolated from dental pulp, periodontal ligament, apical papilla, and gingiva, respectively (Figure 1) [19, 20].

PDLSCs have vital stem cell properties, including high multipotency, great ability for self-renewal, and the ability to express most stem cell markers, i.e., CD166, STRO-1, and CD105 [21]. Hence, the role of PDLSCs could be important in preserving periodontium as well as periodontal regenerative procedures. SCAPs are distinctive stem cells that are promising for endogenous tissue regeneration [22], pulp/dentin regeneration, and bioroot engineering [23]. They are a very unique cell population of postnatal stem cells that are different from DPSCs, in terms of cell motility and migration [24]. This activity will allow the cells to develop into a complex tissue and organ during regeneration; thus, it can be considered as one of the alternative cell resources for neurodegenerative disease therapies. SCAPs have less cellular and vascular components than those in the pulp, and they have osteogenic and dentinogenic potential (because they are mesenchymal stem cells [MSCs]) [25]. A recent study 
from Simonovic and coworkers [26] demonstrated that SCAPs cultured in neurogenic induction medium supplemented with graphene dispersion and water-soluble singlewalled carbon nanotubes exhibit an elevated capacity to differentiate into neural lineage cells. DFSCs come from highly fibrous tissues that are usually extracted and discarded in dental surgery. DFSCs can be cultivated under various culture conditions and thus could be used in tissue engineering and regenerative therapy applications, including neural differentiation [27] and periodontal [20] and tooth root regeneration [28].

SHEDs, PDLSCs, and DFSCs can be obtained from 6- to 12 -year-old individuals [29]. SHEDs possess a great proliferative capacity and the ability to differentiate into adipocytes, neurons, and odontoblast-like cells. They are easily obtained (with minimal or no trauma) due to simple, convenient, and relatively noninvasive techniques [30]. The DPSCs play an important role in tooth homeostasis and remain active throughout life to generate odontoblasts for dentine repair. In vivo, stem cells differentiate according to their specific functions under the action of signaling molecules in a microenvironment called the "stem cell niche." This phenomenon reflects the stem cell native microenvironment, whereby it is thought to preserve the properties and functions of stem cells and monitor differentiation.

DPSCs that are derived from the embryonic cranial neural crest are one of the distinctive types of ecto-MSCs. In the tooth, the DPSCs is located at certain anatomical locations that forming stem cell niches. This niche microenvironment modulates the DPSC populations to promote tissue repair and regeneration [31]. Many signaling molecules in the niche are essential to maintain the stem cell activities, which also have a capacity to regulate cell proliferation and differentiation. DSPCs can differentiate into neural cells to ameliorate nervous system damages [32-34]. They can also differentiate into nonneural cells, including the cartilage [35], bone [36], liver [37], corneal stroma [38], retina [39], and tendon-like tissue [40]. DPSCs can be isolated from dental pulp of third molar teeth without invasive surgery; they are easily cultivated in vitro and expanded for research use. Stem cells harvested from the other tooth regions that involve the infant's exfoliated deciduous immature teeth are known as SHEDs [41]. Many recent updates revealed that DPSCs possess a good proliferative capacity and are multipotential. They can differentiate into neurogenic [42], osteogenic [43], odontogenic [44], and chondrogenic [45] lineages. DPSCs express MSC-like markers (e.g., STRO-1, CD29, CD105, and CD90) [46] and neural stem cell-like markers (e.g., nestin and glial fibrillary acidic protein) [47]; this expression pattern signifies their self-renewal and multipotency capacity. Interestingly, pluripotent stem cell markers such as Oct4, Nanog, Sox, and Klf4 are also regulated by DPSCs. Furthermore, DPSCs have more potent neurogenicity properties and immunosuppressive activities compared to bone marrow mesenchymal stem cells (BMSCs). The abovementioned DPSCs properties make them a strong potential candidate to cure ischemic neurodegenerative disorders [48]. Thus, they could play a significant role in treating neurodegenerative conditions in the human body.
Recent studies reported that secretomes, or conditioned media (CM) acquired from a wide variety of stem cells, can efficiently impede organ damage and ischemic disease. The secretome represents the entire array of proteins and factors that are secreted by a cell into the extracellular space; it constitutes approximately $30 \%$ of the entire proteome in an organism. The secretome contains growth factors, cytokines, chemokines, antibodies, receptors, adhesion molecules, hormones, enzymes, toxins, peptides, proteinases, and antimicrobial peptides. Most of these proteins are actively involved in various biological processes that typically comprise cell attachment [49], proliferation, migration, and differentiation [50], intracellular communication [51], immune response [52], cell survival, and cell defense [53]. All these metabolic and homeostatic processes are essential for the continuity and transformation of life. Previous investigations postulated that DPSC-CM greatly contributes to regenerative therapy, mainly in the CNS [54] and retinal disorders [55]. DPSC-CM offers a therapeutic effect that may implicate a diverse pathway, particularly through the intervening paracrine mechanisms that activate repairing activities. Thus, the repertoire of DPSC-secreted trophic factors might be a significant contributor because it is necessary for neural regeneration.

DSC-CM is useful in enhancing long-term neuronal regeneration in spinal cord injury. DPSC-CM significantly improves cognitive function in a mouse model of $\mathrm{AD}$, specifically by converting the proinflammatory conditions to an anti-inflammatory state. The multifaceted activities offered by SHED-CM may provide neuroprotective effects and could be considered as a potential treatment for the neurodegenerative disorders [56]. A recent study by Yamamoto et al. [57] revealed reduced apoptosis and active proliferation of Schwann cells in the DPSC transplant as opposed to the control conduits. Further in vitro analysis demonstrated that DPSCs promote axon regeneration and stimulate angiogenesis through trophic functions. A study reported by Tsuruta and coworkers [58] showed that systemic SHED-CM administration in a rat subjected to superior laryngeal nerve (SLN) injury successfully improves SLN functional recovery, namely by significantly enhancing axonal regeneration by transforming macrophages to the anti-inflammatory M2 phenotype. It also contributes to angiogenesis at the injured site. Thus, SHED-CM administration may represent an alternative therapeutic option for SLN injury. Considering this evidence, an excellent and noninvasive acellular tool like DPSC-CM and SHED-CM should be further explored for future use in regenerative therapy. In this concise review, we focused on the recent findings using DPSC-CM and SHED-CM for nerve repair, neuroprotection, and neuroregeneration in neurodegenerative diseases and nerve injury.

\section{Methods}

2.1. Search Strategy. This review was systematically conducted by screening all published articles on the effects of the DPSC secretome on nerve regeneration. Two databases were comprehensively used to search for related study (Scopus and PubMed). The keywords used were the combination of dental 


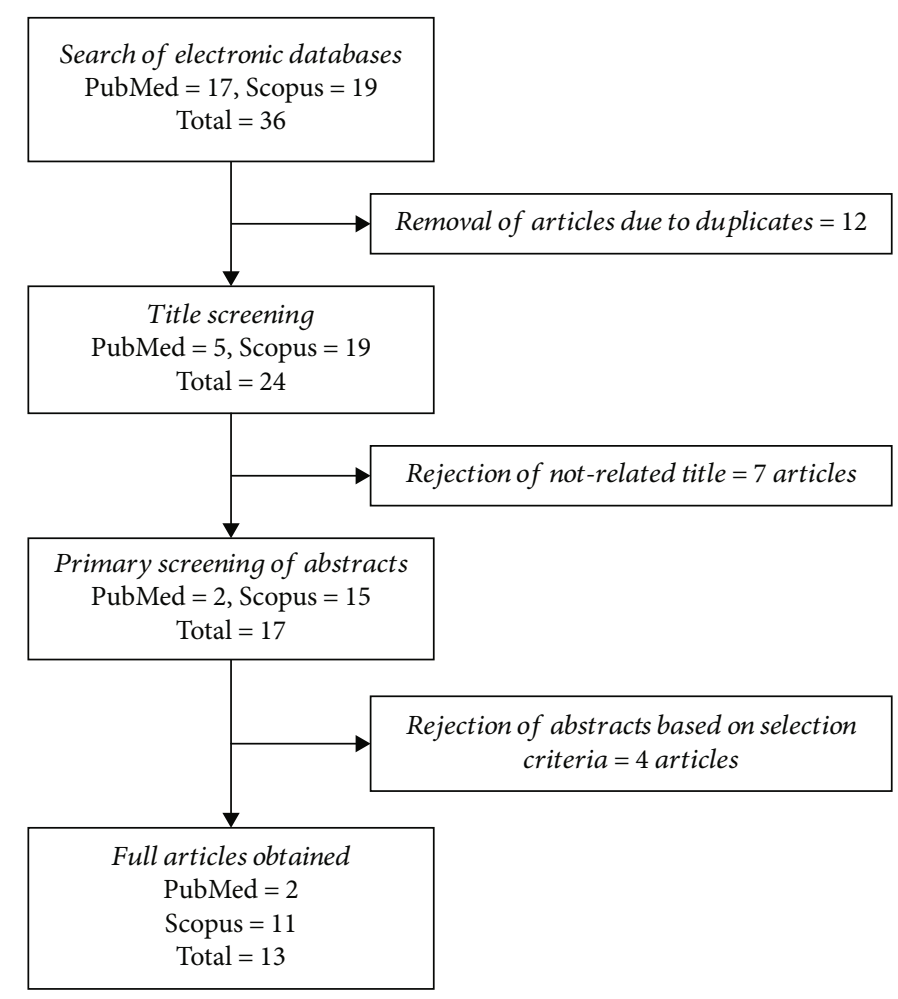

FIgURE 2: Flow chart of the article selection process from PubMed and Scopus and databases.

pulp stem cell secretome OR DPSC secretome OR dental stem cell secretome OR dental pulp stem cell conditioned medium OR DPSC conditioned medium OR dental stem cell conditioned medium AND nerve regeneration OR nerve development OR nerve repair.

2.2. Selection Criteria. Studies published in English from 2000 to 2019 were considered for inclusion. Only articles that provided the full paper were selected. The titles and abstracts were carefully screened to meet the related topic of interest. Primary studies related to the DPSC secretome production, neurotrophic effects, and nerve regeneration potential were included. Only research articles were selected. Review articles, news articles, letters, editorials, and case studies were excluded from the search.

2.3. Data Extraction and Management. All data were extracted from selected articles by two reviewers. The selected papers underwent three screening phases prior to inclusion. The title was first screened for relevance to the topic of interest. Then, the abstracts were carefully screened and unrelated studies were excluded. Lastly, all duplicates were removed. The data were summarized in a table as follows: (1) authors, (2) type of secretome, (3) donor age/condition, (4) type of nerves/disease or cells studied, (5) methodology, (6) passage number/type of medium/period of culture for secretome collection, (7) results, and (8) conclusions.

\section{Results}

3.1. Search Results. Two reviewers independently assessed the articles according to the defined inclusion and exclusion cri- teria. This procedure was performed to minimize bias while selecting articles. At the end of the selection session, a joint discussion was conducted to achieve consensus when differences emerged during the assessment. The primary searches that used the combination of keywords (Section 2.1) only identified 36 articles: 17 from PubMed and 19 from Scopus. Twelve duplicate articles were excluded by title sorting prior to full paper search. After title screening, seven articles were rejected based on the inclusion criteria; these articles were not related to nerve regeneration. Finally, a total of 13 studies were selected for data extraction in this review. The flow chart of the selection process is shown in Figure 2.

3.2. Study Characteristics. The database search provided 13 articles related to DSCs, secretome, CM, nerve regeneration, and neurogenesis. From these articles, various types of dental tissue sources, i.e., SHEDs and adult dental tissue, were used for potential secretome collection/production. SHEDs were extracted from donors aged 6 to 12 years, while adult DSCs came from donors aged 13 to 29 years. The secretome was derived from cells at passage 3 to 9 after 24- to 48-hour culture in serum-free Dulbecco's modified Eagle's medium (DMEM) or minimum essential medium, Eagle alpha modification $(\alpha \mathrm{MEM})$. One study reported the secretome content in detail, with a focus on a set of M2 macrophage inducers (monocyte chemoattractant protein-1 [MCP-1] and secreted ectodomain of sialic acid-binding Ig-like lectin-9 [sSiglec-9]) in the SHED-CM [59]. The remaining articles directly tested the secretome in vitro and in vivo (without prior characterization) to observe their potentiality for neurodifferentiation. One study profiled the secretome from SCAPs [20]. Eight out of thirteen studies conducted in vivo research: 7 in rats 
and 1 in mice. One study reported the therapeutic effects of intravenous administration of the secretome in a rat model [27]. Most studies compared the secretome derived from BMSCs and dental DSCs [20, 27]. All of the studies concluded that the secretome has neurotropic effects on specific nerve repair and regeneration. A summary of the studies is provided in Table 1.

\section{Discussion}

In the past decade, numerous studies reported that MSCs, especially DSCs, can regenerate injured nerves by promoting axonal regeneration and myelin sheath formation. DSCs share a common origin with peripheral nerves and express neuronal markers [27, 32, 33]. Engrafted DSCs alone are susceptible to ischemic attack. However, with appropriate paracrine factors, cells adjacent and distal to the injury site can be influenced to create a unique microenvironment for the stem cells to be functional.

Generally, the secretome includes molecules secreted from cells into the extracellular space; it includes free nucleic acids and soluble proteins and lipids, along with extracellular vesicles (EVs), i.e., microvesicles (MVs) and exosomes that act as intercellular mediators to carry those entities. This broad range of bioactive soluble factors is antiapoptotic, antifibrotic, and anti-inflammatory, and they contain angiogenic regulators, chemoattractive factors, neurotrophic factors (NTFs), and immunomodulators. Stem cells release these molecules through classical and nonclassical secretion mechanisms, including protein translocation, exocytosis, and exosome encapsulation as means of cell-to-cell communication [35].

DPSCs, in particular, secrete various growth factors (GFs) and cytokines. Previous studies revealed high expression levels of transforming growth factor (TGF) and NTFs [60-62]. The NTFs, including nerve growth factor (NGF), glial cell-derived neurotrophic factor (GDNF), neurotrophin-3 (NT-3), brainderived neurotrophic factor (BDNF), ciliary neurotrophic factor (CNTF), vascular endothelial growth factor (VEGF), and hepatocyte growth factor (HGF), are initially necessary for the innervation of dental tissues. Intriguingly, these factors are also vital for the restoration of neural tissues [63].

Neurodegenerative diseases involve brain cell deterioration. In $\mathrm{AD}$, the body starts to produce a protein called amyloid that is deposited as "plaques" in the brain. This phenomenon leads to structural brain changes and consequently prevents the production of neurotransmitters. Previously, alpha-2 macroglobulin (A2M) demonstrated an ability to inhibit amyloid formation [64]. Tachida and colleagues (2015) [60] identified that $\mathrm{A} 2 \mathrm{M}$ is the most prominent secreted protein in the DPSC proteome. A2M is a protease inhibitor and cytokine reporter that might play a key role in the neuroinflammatory response to $\mathrm{AD}$ pathogenesis [65]. A2M can bind to misfolded and aggregation-prone client proteins; this process can mediate the clearance and degradation of $\beta$-amyloid deposits in $\mathrm{AD}$ patients $[66,67]$. Therefore, exogenous A2M derived from the secretome would be expected to at least slow down the progression of brain cell death in AD patients (Figure 3).
Peripheral nerve injury (PNI) is caused by trauma or surgical complications that leads to distal stump demyelination and degradation. Typical symptoms are motor sensory deficits, including weakness, paralysis, and pain. Current treatments, such as direct repair and autologous nerve grafts, are still insufficient. Recently, Tsuruta and colleagues (2018) [58] reported that the DPSC-derived secretome can induce neuronal regeneration. Based on their findings, the authors suggested that the systemic administration of SHED-CM may provide therapeutic benefits in PNI treatment. As previously reported by Sugimura-Wakayama and coworkers (2015) [68], the SHED-CM secretome contains NGF, BDNF, NT-3, CNTF, and GDNF, all of which create a more desirable extracellular microenvironment for peripheral nerve regeneration [34]. They reported that SHED-CM enhances migration and proliferation in vitro. It also promotes axonal regeneration and functional recovery in a sciatic nerve defect rat, including enhanced axon growth, angiogenesis, migration, proliferation, and neuron survival. This data indicate that SHED-CM contains factors that can regulate the mobilization of Schwann cells to the target tissue. Thus, it serves as a potential PNI treatment [34].

Macrophages involved in distal degeneration can promote the switch from the proinflammatory (M1) to the anti-inflammatory (M2) phenotype. A set of tissuerepairing M2 macrophage inducers, i.e., sSiglec-9 and MCP-1, enhance nerve regeneration $[29,69]$. SHED-CM that contains MCP-1 and sSiglec-9 enhances neurite extension of the peripheral nerve, data that suggest these factors can promote the formation of a Schwann cell bridge and axonal extension. The depletion of both MCP-1 and sSiglec-9 in SHED-CM reduces its ability to restore neurological function and to regenerate peripheral nerves [29]. On the other hand, a study by Matsubara and colleagues identified the M2 inducers activate multifaceted endogenous neurorepair mechanisms, effects that restore locomotor function in a rat model of spinal cord injury (SCI). M2 inducers directly convert the proinflammatory conditions prevalent in the damaged CNS to tissue-repairing function by modulating the microglia/macrophage phenotype (Figure 4) [69].

Other than NTFs, El-Moataz and colleagues (2016) [70] revealed that the DPSC secretome contains a high concentration of cytokines, including fractalkine (FKN), which is regulated on activation and normally $\mathrm{T}$ cell expressed, and presumably secreted RANTES and FMS-like tyrosine kinase 3 (FLT-3). FKN, also known as chemokine (C-X3-C motif) ligand 1 (CXCL1), promotes microglia survival under neurotoxic conditions and enhances the ability of macrophages and microglia to execute their phagocytic functions. The activation of the phagocytic response is important to clear cellular debris and stress-response pathways to counteract any remaining neurotoxic molecules that caused the initial damage, especially in neurodegenerative diseases (Figure 3) [71]. On the other hand, RANTES, also known as chemokine (C-C motif) ligand 5 (CCL5), aids during acute infection by promoting macrophage infiltration, mobilization, and function at injured sites. FLT-3 is involved in differentiation, proliferation, and survival of dendritic cells. RANTES and FLT3 are both implicated in the processing of pain 


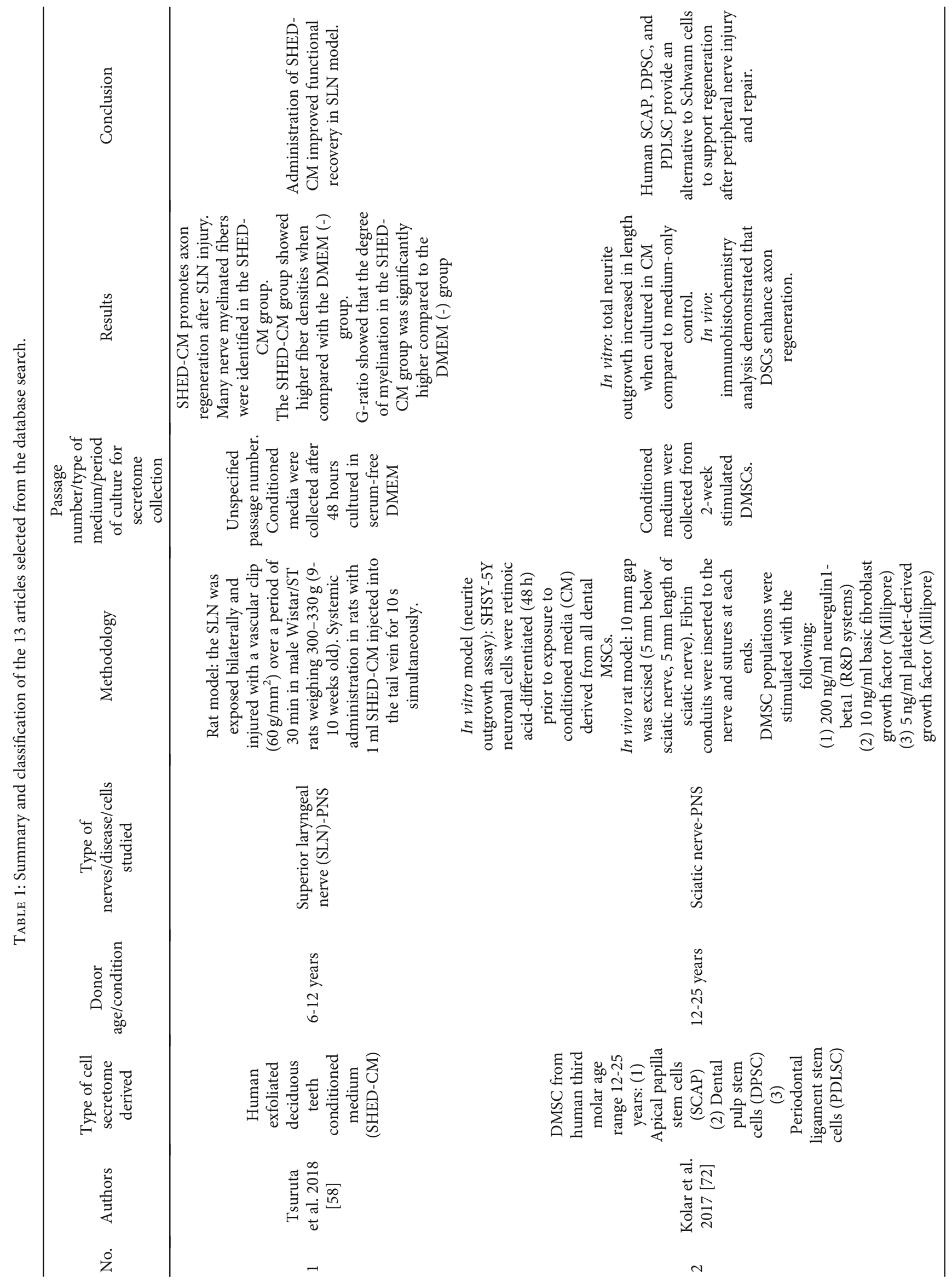




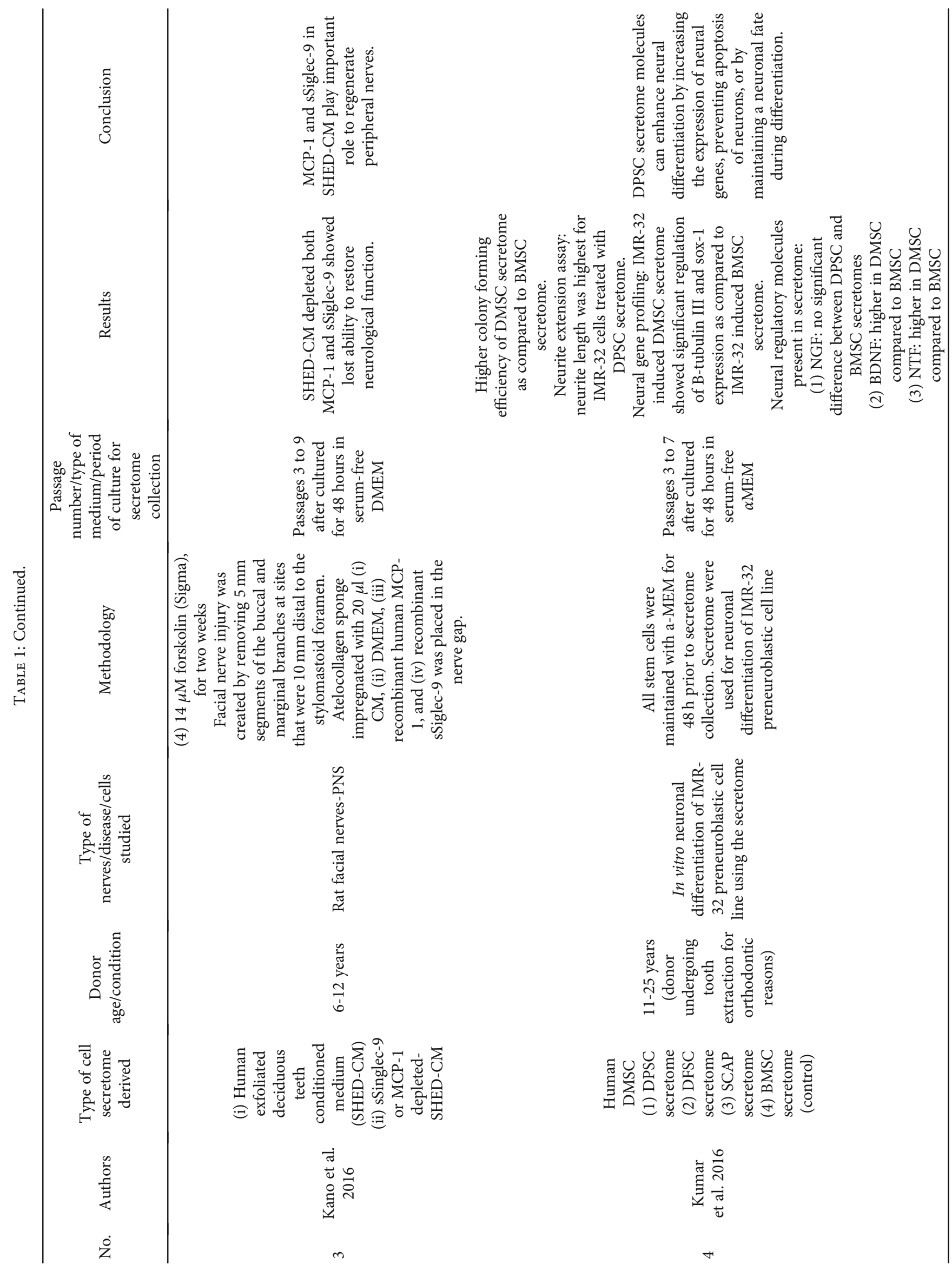




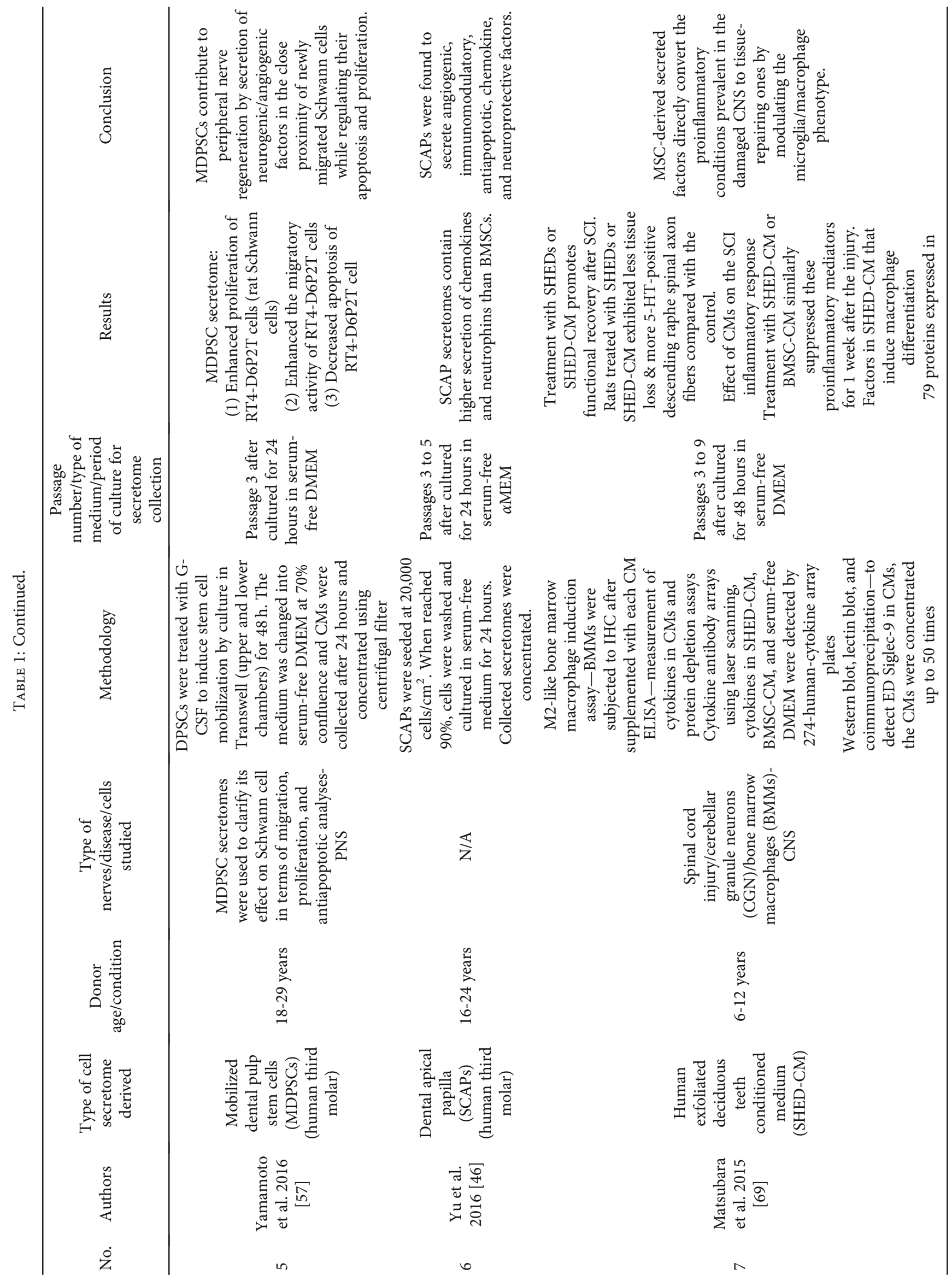




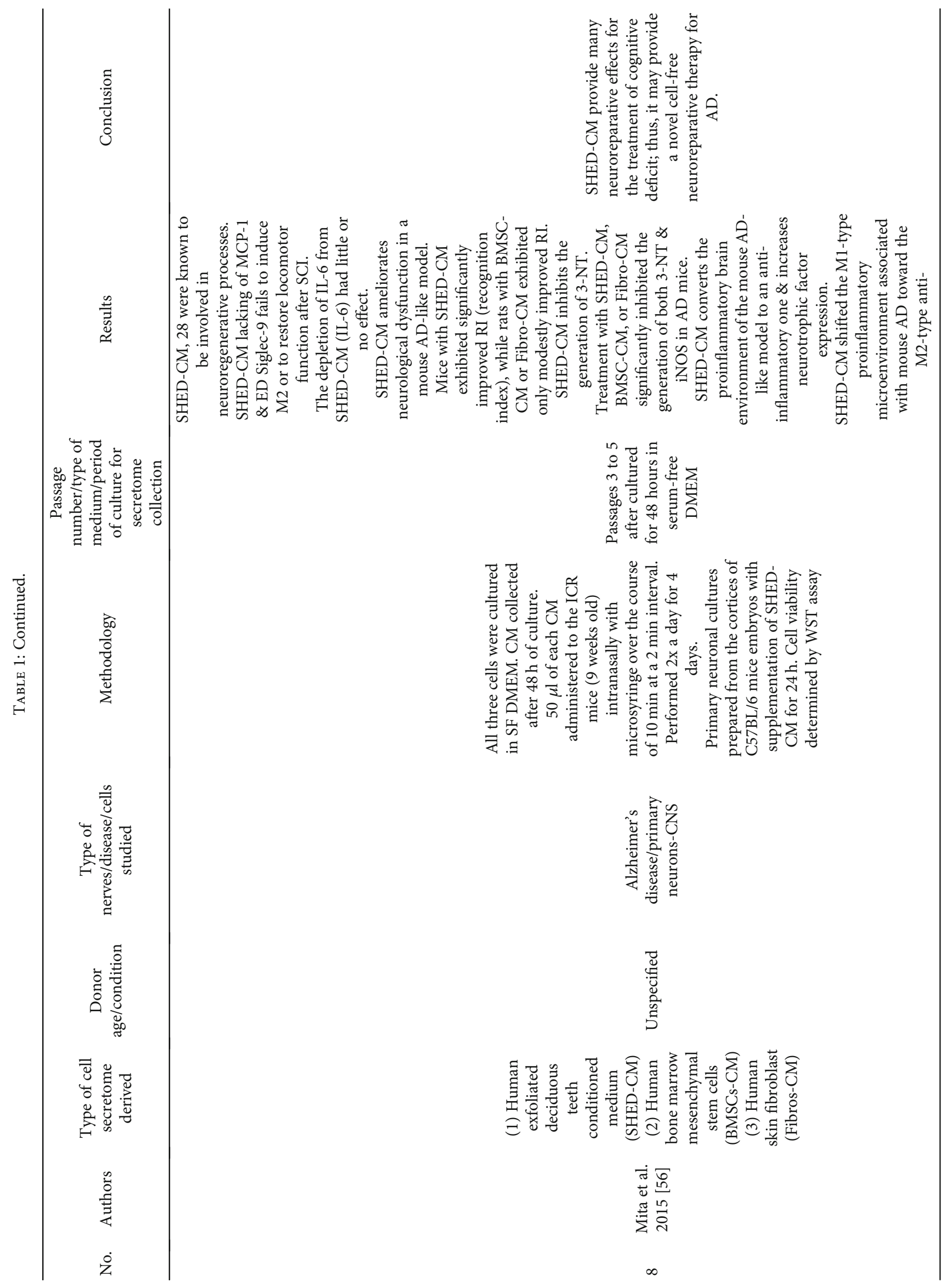




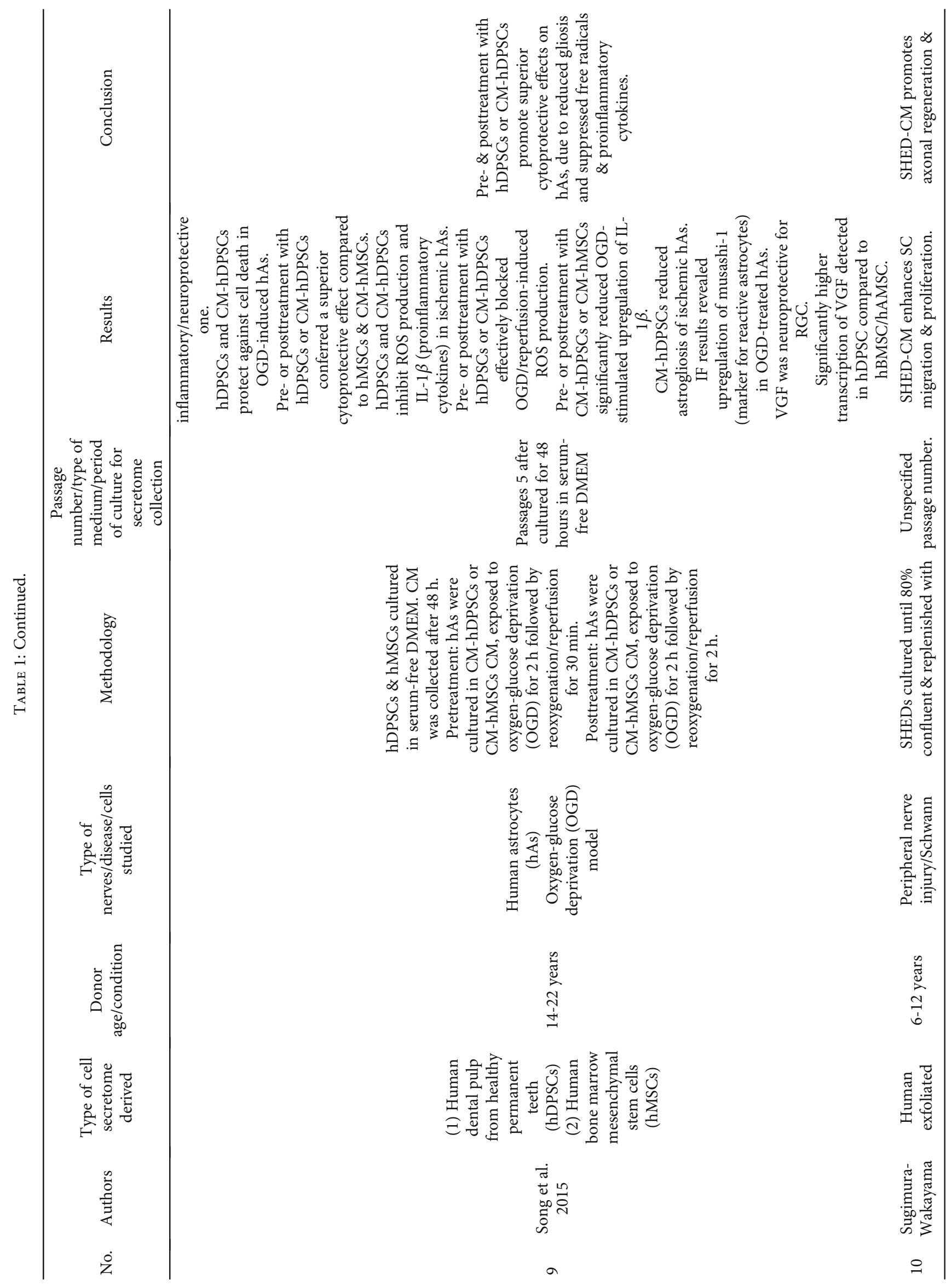




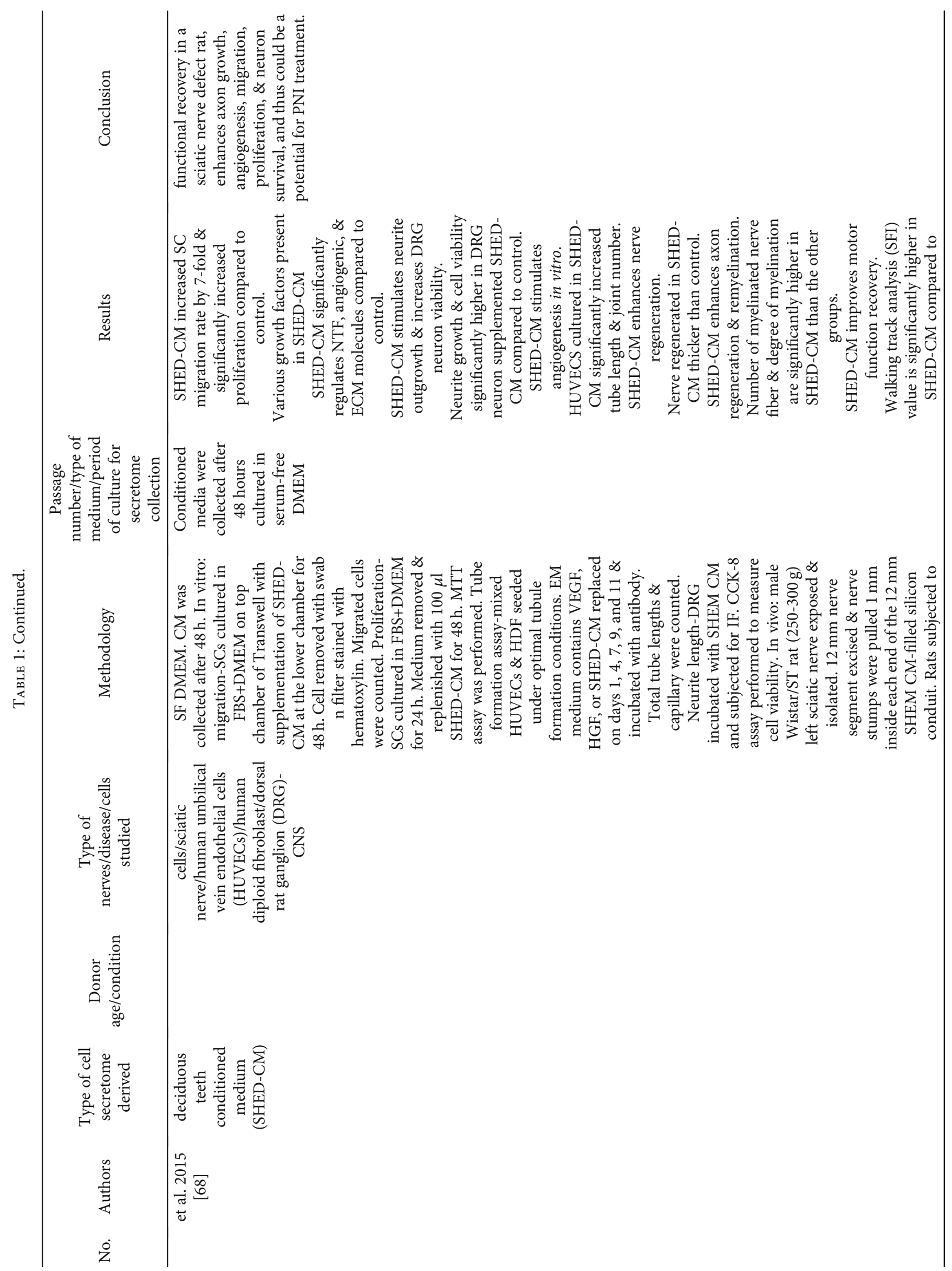




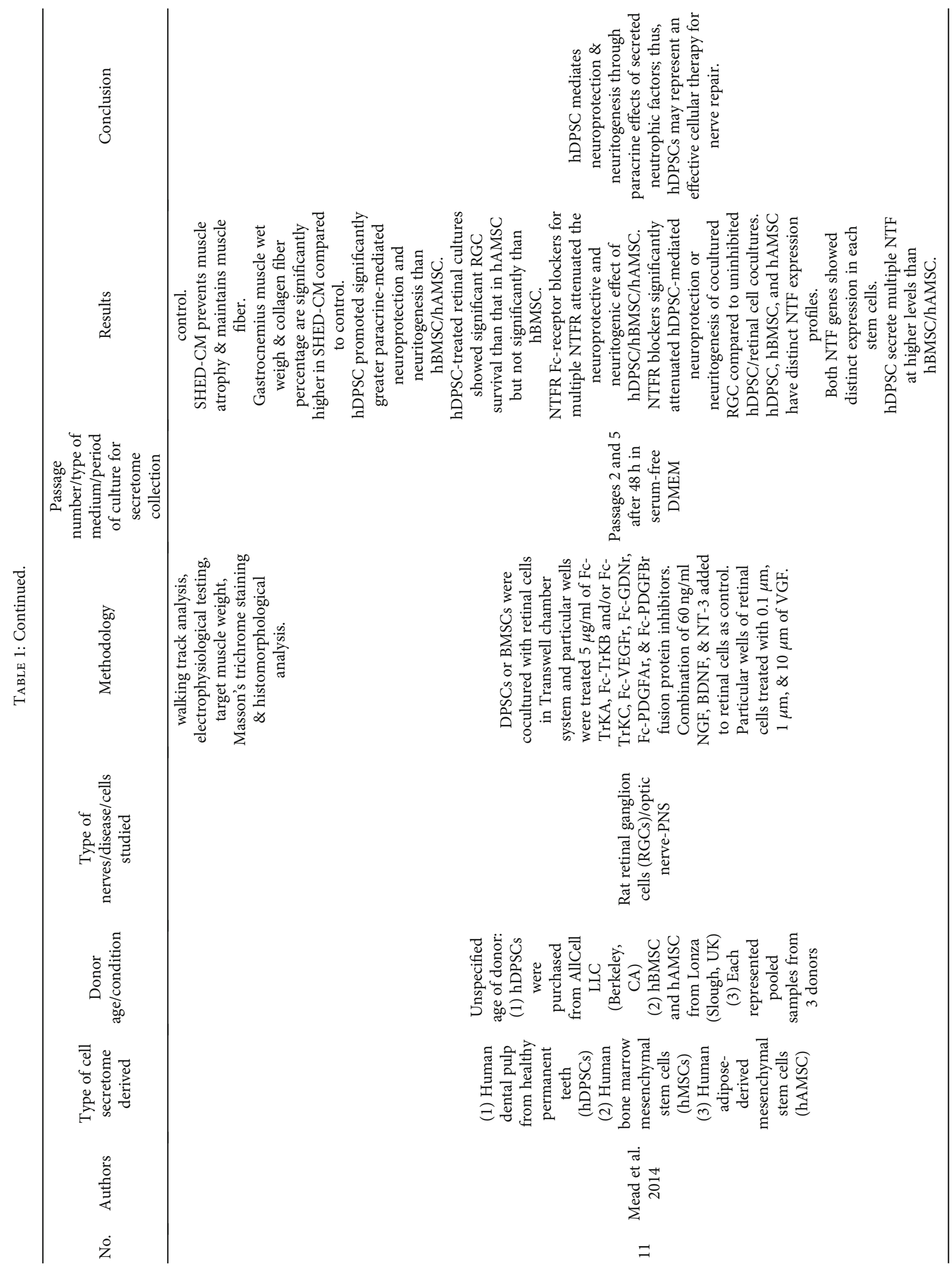




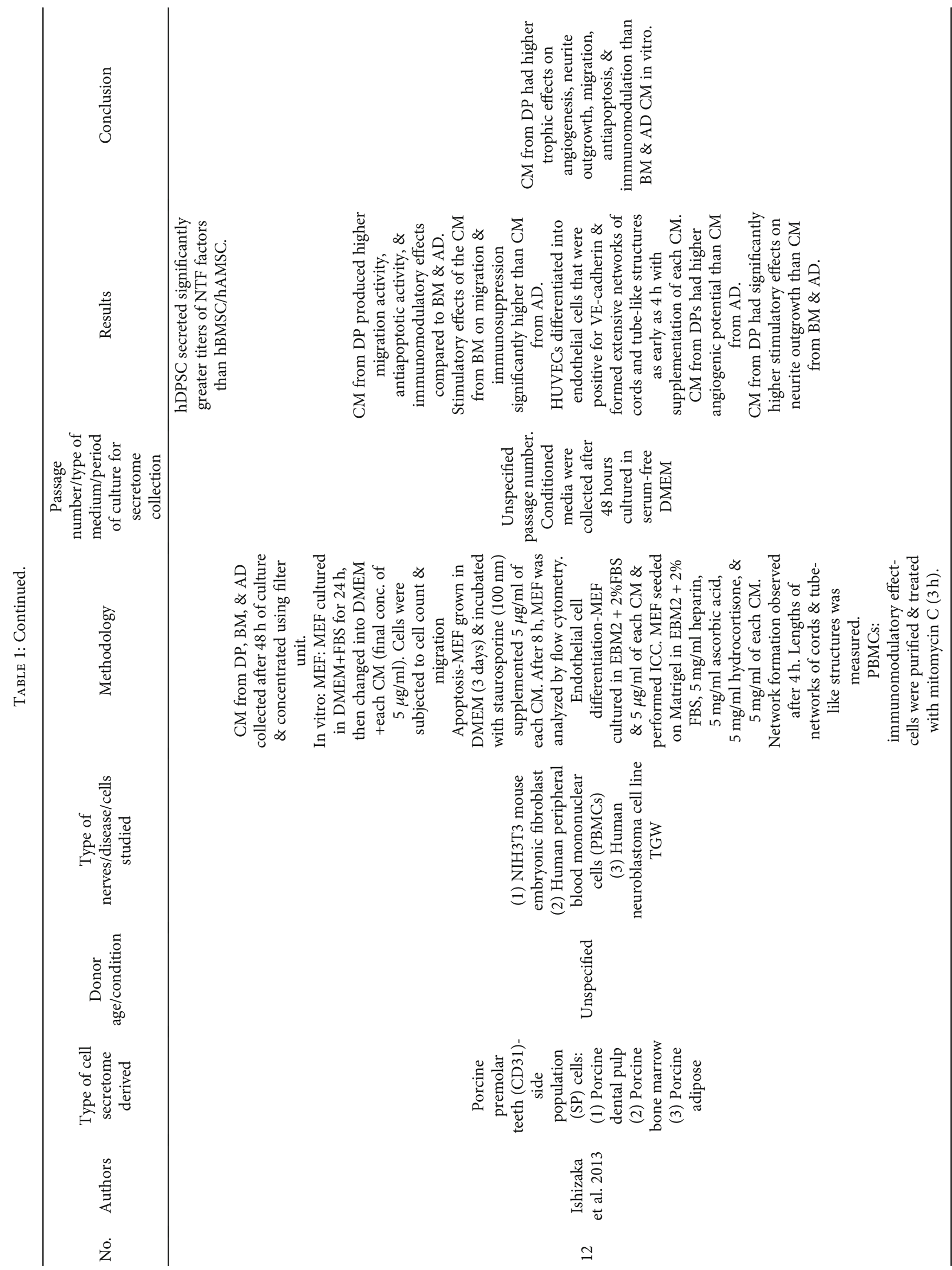




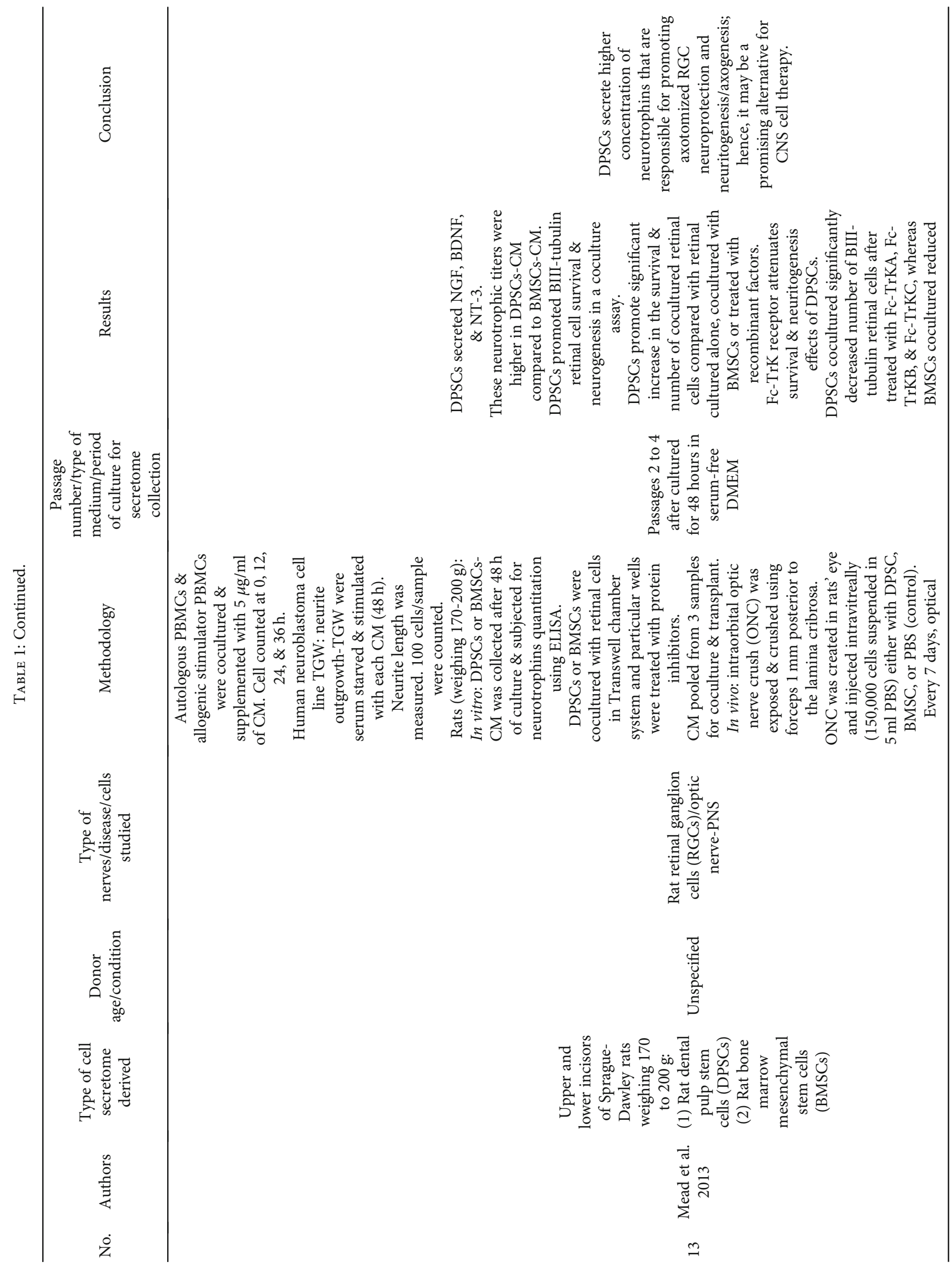




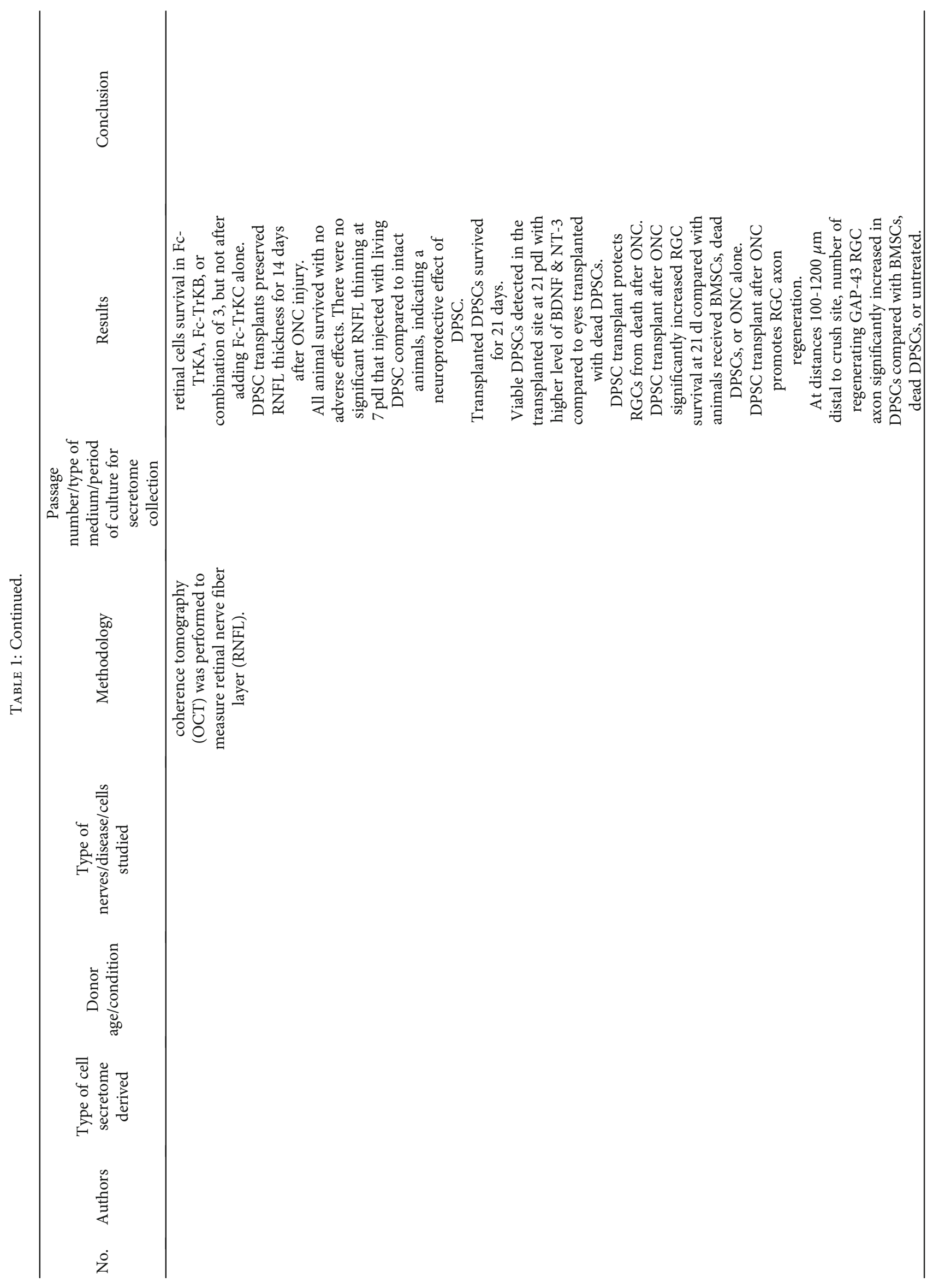




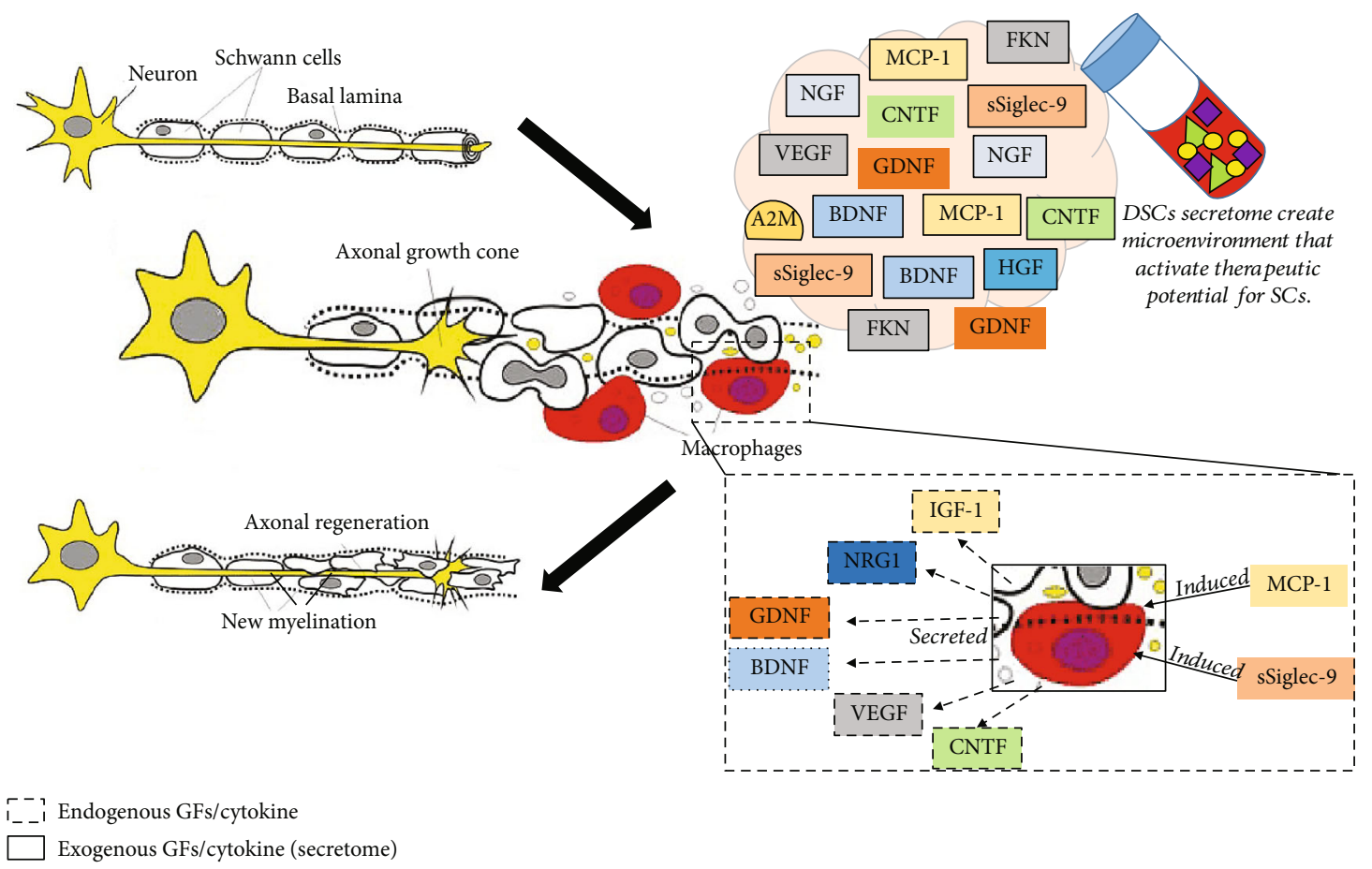

FIGURE 3: Schematic illustrating the role of DSC secretomes modulating the nerve regeneration in CNS. Alzheimer's disease (AD) responds to the production of $\beta$-amyloid fibres/plaque which triggers the microglia and astrocytes activation and generation of proinflammatory cytokines. The chronic activation of microglia and astrocytes causes neuron degeneration. Stimulation by GFs and cytokine derived from DSCs secretome such as A2M cytokine is capable of binding to $\beta$-amyloid fibres/plaque that mediate the clearance and degradation [1] while FKN can execute their phagocytic functions. In addition, Siglec-9 and MCP-1 can switch the M1 to M2 phenotype for nerve regeneration. This would enhance neuronal plasticity and neurogenesis in $\mathrm{AD}$ patients.

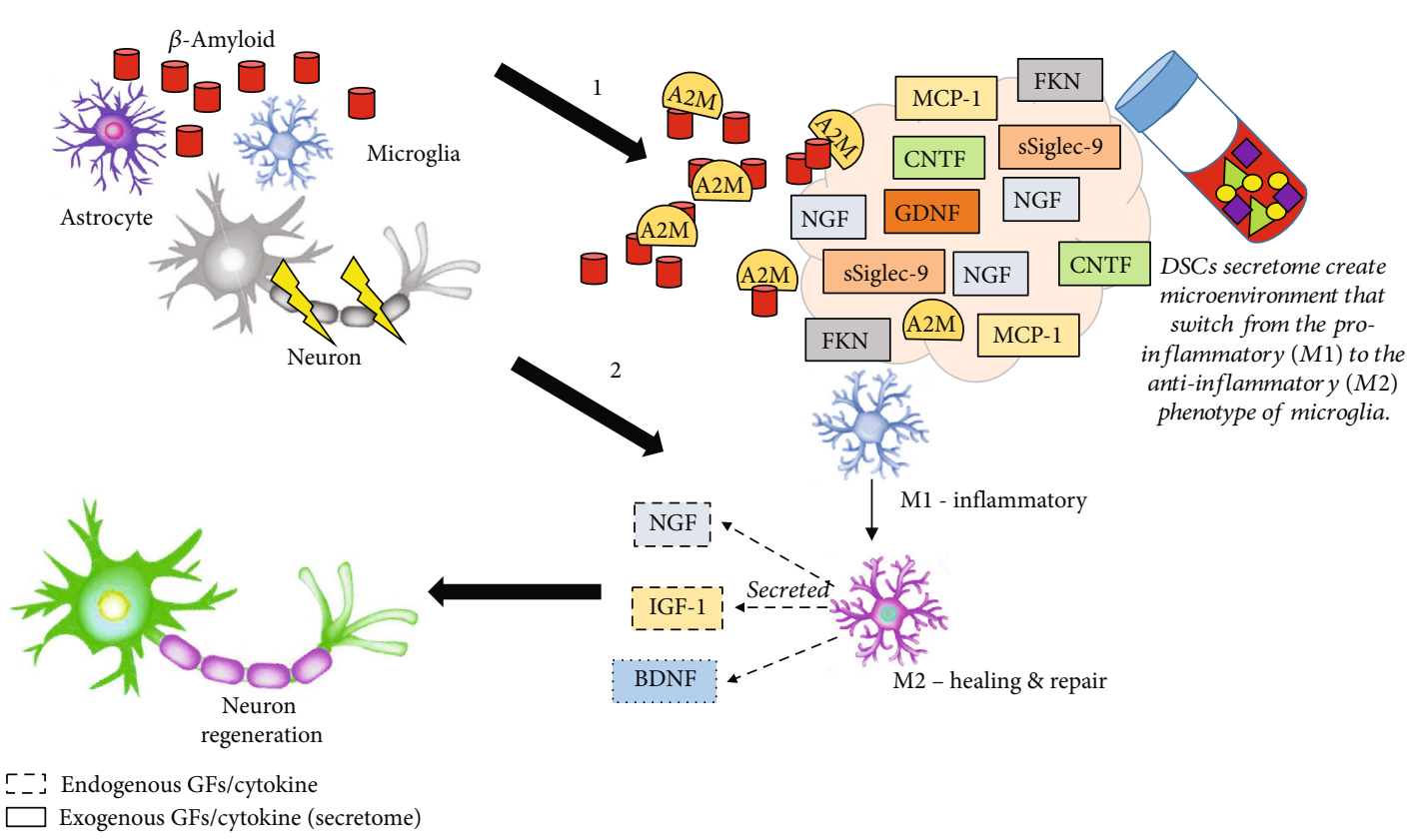

FIGURE 4: Schematic illustrating the role of DSC secretomes modulating the nerve regeneration in PNS. Axon of the neuron is myelinated by the Schwann cells (SCs). Nerve injury has caused the myelin sheaths and axon degenerated. There are series of macrophage activation by several GFs and cytokine involved to remove the debris of degenerating fibers like A2M cytokine that recognized by specific receptor on macrophages. On the other hand, MCP-1 and sSiglec-9 induced the polarization of M2 macrophage. This has caused MCP-1/sSiglec-9induced M2 macrophages expressed six factors that are known to affect the functional properties of SCs. The six factors are IGF-1, NRG1, BDNF, CNTF, VEGF and GDNF. These factors promote the proliferation, migration, and differentiation of SCs that can enhance axonal regeneration. Neuron image adopted and modified from Mey et al. [75]. 
information in peripheral nociception. However, direct evidence and the possible mechanism of this action is lacking.

Collectively, the secretomes derived from SHEDs and DPSCs demonstrate the most potential for nerve regeneration. Besides having a high proliferation rate, DPSCs also exhibit more growth factors and cytokines compared to other MSCs (e.g., BMSCs) [38]. The DSC-CM contains complex soluble signaling molecules and growth factors that can create a potent odontogenic microenvironment, and the paracrine mechanism of release makes them attractive for use in neuroregeneration [39].

Another aspect to consider is the method used to collect the CM. Cell confluency, passage number, incubation time, growth medium, and induction are crucial aspects to ensure that cells secrete distinct, beneficial proteins. DSCs stimulated by specific GFs, including neuregulin, basic fibroblast growth factor (bFGF), platelet-derived growth factor (PDGF), and forskolin, provide an alternative to Schwann cells to support regeneration after PNI $[40,72]$. It is also worthy to compare the common two-dimensional cell culture technique with a three-dimensional culture. Previously, three-dimensional BMSC cultures were reported to produce significantly more secretome [41]. However, it still remains unknown whether the three-dimensional DPSC secretome contains "extra" growth factors and signaling molecules to exert their beneficial effects for better execution of neuroregeneration.

Cell-based therapy limitations led scientists to find a new method that can deliver therapeutic value to the patient. It is well-documented that the DSC secretome contains various growth factors and cytokines along with EVs that act as a stable cargo. EVs can be transported into cells via endocytosis. EVs can also penetrate the bloodbrain barrier, a crucial feature given that many drugs may not be able to penetrate it [73]. However, the composition of cargo in EVs depends on cell type and culture and induction conditions. Thus, it is very important to identify the cargo in EVs prior to their use for a specific treatment. Therefore, secretome generation must be properly performed with good manufacturing practice (GMP) to achieve a clinical-grade product prior to its use as a treatment in clinical applications.

\section{Conflicts of Interest}

The authors declare that there is no conflict of interest regarding the publication of this paper.

\section{Acknowledgments}

The research was performed with the financial support of Universiti Kebangsaan Malaysia (GGPM-2018-009).

\section{References}

[1] J. Stephenson, E. Nutma, P. van der Valk, and S. Amor, "Inflammation in CNS neurodegenerative diseases," Immunology, vol. 154, no. 2, pp. 204-219, 2018.

[2] A. U. Bayer, O. N. Keller, F. Ferrari, and K. P. Maag, "Association of glaucoma with neurodegenerative diseases with apoptotic cell death: Alzheimer's disease and Parkinson's disease," American Journal of Ophthalmology, vol. 133, no. 1, pp. 135-137, 2002.

[3] E. Deas, N. Cremades, P. R. Angelova et al., "Alpha-synuclein oligomers interact with metal ions to induce oxidative stress and neuronal death in Parkinson's disease," Antioxidants \& Redox Signaling, vol. 24, no. 7, pp. 376-391, 2016.

[4] S. B. Prusiner, "Shattuck lecture - neurodegenerative diseases and prions," The New England Journal of Medicine, vol. 344, no. 20, pp. 1516-1526, 2001.

[5] N. A. Simonian and J. T. Coyle, "Oxidative stress in neurodegenerative diseases," Annual Review of Pharmacology and Toxicology, vol. 36, no. 1, pp. 83-106, 1996.

[6] D. F. Preisig, L. Kulic, M. Krüger et al., "High-speed video gait analysis reveals early and characteristic locomotor phenotypes in mouse models of neurodegenerative movement disorders," Behavioural Brain Research, vol. 311, pp. 340-353, 2016.

[7] R. B. Postuma and D. Berg, "The New Diagnostic Criteria for Parkinson's Disease," in International Review of Neurobiology, Academic Press, New York, 2017.

[8] R. Savica, B. R. Grossardt, W. A. Rocca, and J. H. Bower, "Parkinson disease with and without dementia: a prevalence study and future projections," Movement Disorders, vol. 33, no. 4, pp. 537-543, 2018.

[9] J. Gaugler, B. James, T. Johnson, K. Scholz, and J. Weuve, "2016 Alzheimer's disease facts and figures," Alzheimer's \& Dementia, vol. 12, no. 4, pp. 459-509, 2016.

[10] T. A. Mestre and K. Shannon, "Huntington disease care: from the past to the present, to the future," Parkinsonism \& Related Disorders, vol. 44, pp. 114-118, 2017.

[11] M. D. Rawlins, N. S. Wexler, A. R. Wexler et al., "The prevalence of Huntington's disease," Neuroepidemiology, vol. 46, no. 2, pp. 144-153, 2016.

[12] M. Chin-Chan, J. Navarro-Yepes, and B. Quintanilla-Vega, "Environmental pollutants as risk factors for neurodegenerative disorders: Alzheimer and Parkinson diseases," Frontiers in Cellular Neuroscience, vol. 9, 2015.

[13] J. L. Robinson, E. B. Lee, S. X. Xie et al., "Neurodegenerative disease concomitant proteinopathies are prevalent, agerelated and APOE4-associated," Brain, vol. 141, no. 7, pp. 2181-2193, 2018.

[14] J. Paloczi, Z. V. Varga, G. Hasko, and P. Pacher, "Neuroprotection in oxidative stress-related neurodegenerative diseases: role of endocannabinoid system modulation," Antioxidants and Redox Signaling, vol. 29, no. 1, pp. 75-108, 2018.

[15] M. Whiteside, J. Marvin Herndon, S. Borrego Alonso, and C. E. LJ, "(3) review article Whiteside and Herndon," Journal of Advances in Medicine and Medical Research, vol. 25, no. 10, pp. 1-11, 2018.

[16] Y. C. Xue, R. Feuer, N. Cashman, and H. Luo, "Enteroviral infection: the forgotten link to amyotrophic lateral sclerosis?," Frontiers in Molecular Neuroscience, vol. 11, 2018.

[17] A. Kulshreshtha and P. Piplani, "Current pharmacotherapy and putative disease-modifying therapy for Alzheimer's disease," Neurological Sciences, vol. 37, no. 9, pp. 1403-1435, 2016.

[18] R. Manenti, M. Brambilla, A. Benussi et al., "Mild cognitive impairment in Parkinson's disease is improved by transcranial direct current stimulation combined with physical therapy," Movement Disorders, vol. 31, no. 5, pp. 715-724, 2016.

[19] P. T. Sharpe, "Dental mesenchymal stem cells," Development, vol. 143, no. 13, pp. 2273-2280, 2016. 
[20] P. M. Bartold, S. Shi, and S. Gronthos, "Stem cells and periodontal regeneration," Periodontology 2000, vol. 40, no. 1, pp. 164-172, 2006.

[21] K. Nagatomo, M. Komaki, I. Sekiya et al., "Stem cell properties of human periodontal ligament cells," Journal of Periodontal Research, vol. 41, no. 4, pp. 303-310, 2006.

[22] O. A. Nada and R. M. El Backly, "Stem cells from the apical papilla (SCAP) as a tool for endogenous tissue regeneration," Frontiers in Bioengineering and Biotechnology, vol. 6, 2018.

[23] G. T. J. Huang, W. Sonoyama, Y. Liu, H. Liu, S. Wang, and S. Shi, "The hidden treasure in apical papilla: the potential role in pulp/dentin regeneration and bioroot engineering," Journal of Endodontics, vol. 34, no. 6, pp. 645-651, 2008.

[24] W. Sonoyama, Y. Liu, D. Fang et al., "Mesenchymal stem cellmediated functional tooth regeneration in swine," PLoS One, vol. 1, no. 1, 2006.

[25] W. Sonoyama, Y. Liu, T. Yamaza et al., "Characterization of the apical papilla and its residing stem cells from human immature permanent teeth: a pilot study," Journal of Endodontia, vol. 34, no. 2, pp. 166-171, 2008.

[26] J. Simonovic, B. Toljic, N. Nikolic et al., "Differentiation of stem cells from apical papilla into neural lineage using graphene dispersion and single walled carbon nanotubes," Journal of Biomedical Materials Research Part A, vol. 106, no. 10, pp. 2653-2661, 2018.

[27] C. Morsczeck, F. Völlner, M. Saugspier et al., "Comparison of human dental follicle cells (DFCs) and stem cells from human exfoliated deciduous teeth (SHED) after neural differentiation in vitro," Clinical Oral Investigations, vol. 14, no. 4, pp. 433440, 2010.

[28] B. Yang, G. Chen, J. Li et al., "Tooth root regeneration using dental follicle cell sheets in combination with a dentin matrix - based scaffold," Biomaterials, vol. 33, no. 8, pp. 2449-2461, 2012.

[29] F. Kano, K. Matsubara, M. Ueda, H. Hibi, and A. Yamamoto, "Secreted ectodomain of sialic acid-binding Ig-like lectin-9 and monocyte chemoattractant protein-1 synergistically regenerate transected rat peripheral nerves by altering macrophage polarity," Stem Cells, vol. 35, no. 3, pp. 641-653, 2017.

[30] V. Arora, P. Arora, and A. Munshi, "Banking stem cells from human exfoliated deciduous teeth (SHED): saving for the future," The Journal of Clinical Pediatric Dentistry, vol. 33, no. 4, pp. 289-294, 2009.

[31] T. A. Mitsiadis, A. Feki, G. Papaccio, and J. Catón, "Dental pulp stem cells, niches, and notch signaling in tooth injury," Advances in Dental Research, vol. 23, no. 3, pp. 275-279, 2011.

[32] K. Sanen, W. Martens, M. Georgiou, M. Ameloot, I. Lambrichts, and J. Phillips, "Engineered neural tissue with Schwann cell differentiated human dental pulp stem cells: potential for peripheral nerve repair?," Journal of Tissue Engineering and Regenerative Medicine, vol. 11, no. 12, pp. 3362-3372, 2017.

[33] S. Das and J. R. Bellare, "Dental pulp stem cells in customized 3D nanofibrous scaffolds for regeneration of peripheral nervous system," in Methods in Molecular Biology, Humana Press, New York, 2018.

[34] J. Zhang, M. Lian, P. Cao et al., "Effects of nerve growth factor and basic fibroblast growth factor promote human dental pulp stem cells to neural differentiation," Neurochemical Research, vol. 42, no. 4, pp. 1015-1025, 2017.
[35] M. Mata, L. Milian, M. Oliver et al., "In vivo_ articular cartilage regeneration using human dental pulp stem cells cultured in an alginate scaffold: a preliminary study," Stem Cells International, vol. 2017, Article ID 8309256, 9 pages, 2017.

[36] A. L. Junior, C. C. G. Pinheiro, T. L. Fernandes, and D. F. Bueno, "The use of human dental pulp stem cells for in vivo bone tissue engineering: a systematic review," Journal of Tissue Engineering, vol. 9, 2018.

[37] S. Ohkoshi, H. Hirono, T. Nakahara, and H. Ishikawa, "Dental pulp cell bank as a possible future source of individual hepatocytes," World Journal of Hepatology, vol. 10, no. 10, pp. 702707, 2018.

[38] F. N. Syed-Picard, Y. Du, K. L. Lathrop, M. M. Mann, M. L. Funderburgh, and J. L. Funderburgh, "Dental pulp stem cells: a new cellular resource for corneal stromal regeneration," Stem Cells Translational Medicine, vol. 4, no. 3, pp. 276-285, 2015.

[39] R. Roozafzoon, A. Lashay, M. Vasei et al., "Dental pulp stem cells differentiation into retinal ganglion-like cells in a three dimensional network," Biochemical and Biophysical Research Communications, vol. 457, no. 2, pp. 154-160, 2015.

[40] Y. Y. Chen, S. T. He, F. H. Yan et al., "Dental pulp stem cells express tendon markers under mechanical loading and are a potential cell source for tissue engineering of tendon-like tissue," International Journal of Oral Science, vol. 8, no. 4, pp. 213-222, 2016.

[41] P. D. Potdar, "Human dental pulp stem cells: applications in future regenerative medicine," World Journal of Stem Cells, vol. 7, no. 5, pp. 839-851, 2015.

[42] S. Y. Chun, S. Soker, Y. J. Jang, T. G. Kwon, and E. S. Yoo, “Differentiation of human dental pulp stem cells into dopaminergic neuron-like cells in vitro," Journal of Korean Medical Science, vol. 31, no. 2, pp. 171-177, 2016.

[43] T. Yasui, Y. Mabuchi, H. Toriumi et al., "Purified human dental pulp stem cells promote osteogenic regeneration," Journal of Dental Research, vol. 95, no. 2, pp. 206-214, 2016.

[44] F. Paduano, M. Marrelli, L. J. White, K. M. Shakesheff, and M. Tatullo, "Odontogenic differentiation of human dental pulp stem cells on hydrogel scaffolds derived from decellularized bone extracellular matrix and collagen type I," PLOS One, vol. 11, no. 2, 2016.

[45] L. Yao and N. Flynn, "Dental pulp stem cell-derived chondrogenic cells demonstrate differential cell motility in type I and type II collagen hydrogels," The Spine Journal, vol. 18, no. 6, pp. 1070-1080, 2018.

[46] N. Homayounfar, P. Verma, A. Nosrat et al., "Isolation, characterization, and differentiation of dental pulp stem cells in ferrets," Journal of Endodontia, vol. 42, no. 3, pp. 418-424, 2016.

[47] Y. W. Geng, Z. Zhang, M. Y. Liu, and W. P. Hu, "Differentiation of human dental pulp stem cells into neuronal by resveratrol," Cell Biology International, vol. 41, no. 12, pp. 1391$1398,2017$.

[48] C. Nito, K. Sowa, M. Nakajima et al., "Transplantation of human dental pulp stem cells ameliorates brain damage following acute cerebral ischemia," Biomedicine \& Pharmacotherapy, vol. 108, pp. 1005-1014, 2018.

[49] E. G. Hayman, M. D. Pierschbacher, S. Suzuki, and E. Ruoslahti, "Vitronectin-a major cell attachmentpromoting protein in fetal bovine serum," Experimental Cell Research, vol. 160, no. 2, pp. 245-258, 1985.

[50] M. L. Kireeva, F. E. Mo, G. P. Yang, and L. F. Lau, “Cyr61, a product of a growth factor-inducible immediate-early gene, 
promotes cell proliferation, migration, and adhesion," Molecular and Cellular Biology, vol. 16, no. 4, pp. 1326-1334, 1996.

[51] K. Y. Leung, B. A. Siame, H. Snowball, and Y. K. Mok, “Type VI secretion regulation: crosstalk and intracellular communication," Current Opinion in Microbiology, vol. 14, no. 1, pp. 9-15, 2011.

[52] J. P. Hewitson, J. R. Grainger, and R. M. Maizels, "Helminth immunoregulation: the role of parasite secreted proteins in modulating host immunity," Molecular and Biochemical Parasitology, vol. 167, no. 1, pp. 1-11, 2009.

[53] I. M. Copple, C. E. Goldring, N. R. Kitteringham, and B. K. Park, "The Keap1-Nrf2 cellular defense pathway: mechanisms of regulation and role in protection against drug-induced toxicity," in Handbook of Experimental Pharmacology, vol. 196, Springer, Berlin, Heidelberg, 2010.

[54] M. Omi, M. Hata, N. Nakamura et al., "Transplantation of dental pulp stem cells suppressed inflammation in sciatic nerves by promoting macrophage polarization towards antiinflammation phenotypes and ameliorated diabetic polyneuropathy," Journal of Diabetes Investigation, vol. 7, no. 4, pp. 485-496, 2016.

[55] B. Mead, A. Logan, M. Berry, W. Leadbeater, and B. A. Scheven, "Concise review: dental pulp stem cells: a novel cell therapy for retinal and central nervous system repair," Stem Cells, vol. 35, no. 1, pp. 61-67, 2017.

[56] T. Mita, Y. Furukawa-Hibi, H. Takeuchi et al., "Conditioned medium from the stem cells of human dental pulp improves cognitive function in a mouse model of Alzheimer's disease," Behavioural Brain Research, vol. 293, pp. 189-197, 2015.

[57] T. Yamamoto, Y. Osako, M. Ito et al., “Trophic effects of dental pulp stem cells on Schwann cells in peripheral nerve regeneration," Cell Transplantation, vol. 25, no. 1, pp. 183-193, 2016.

[58] T. Tsuruta, K. Sakai, J. Watanabe, W. Katagiri, and H. Hibi, "Dental pulp-derived stem cell conditioned medium to regenerate peripheral nerves in a novel animal model of dysphagia," PLoS One, vol. 13, no. 12, p. e0208938, 2018.

[59] B. M. Seo, M. Miura, S. Gronthos et al., "Investigation of multipotent postnatal stem cells from human periodontal ligament," Lancet, vol. 364, no. 9429, pp. 149-155, 2004.

[60] Y. Tachida, H. Sakurai, and J. Okutsu, "Proteomic comparison of the secreted factors of mesenchymal stem cells from bone marrow, adipose tissue and dental pulp," Journal of Proteomics \& Bioinformatics, vol. 8, no. 12, 2015.

[61] A. J. Salgado, J. C. Sousa, B. M. Costa et al., "Mesenchymal stem cells secretome as a modulator of the neurogenic niche: basic insights and therapeutic opportunities," Frontiers in Cellular Neuroscience, vol. 9, 2015.

[62] A. Kumar, V. Kumar, V. Rattan, V. Jha, and S. Bhattacharyya, "Secretome cues modulate the neurogenic potential of bone marrow and dental stem cells," Molecular Neurobiology, vol. 54, no. 6, pp. 4672-4682, 2017.

[63] F. Asutay, A. H. Acar, Ü. Yolcu, M. Kirtay, and H. Alan, "Dental stem cell sources and their potentials for bone tissue engineering," Journal of Istanbul University Faculty of Dentistry, vol. 49, no. 2, pp. 51-56, 2019.

[64] A. R. Wyatt, P. Constantinescu, H. Ecroyd et al., "Protease-activated alpha-2-macroglobulin can inhibit amyloid formation via two distinct mechanisms," FEBS Letters, vol. 587, no. 5, pp. 398-403, 2013.

[65] J. Bauer, S. Strauss, U. Schreiter-Gasser et al., "Interleukin-6 and $\alpha$-2-macroglobulin indicate an acute-phase state in Alz- heimer's disease cortices," FEBS Letters, vol. 285, no. 1, pp. 111-114, 1991.

[66] K. French, J. J. Yerbury, and M. R. Wilson, "Protease activation of $\alpha 2$-macroglobulin modulates a chaperone-like action with broad specificity," Biochemistry, vol. 47, no. 4, pp. 1176$1185,2008$.

[67] V. R. Varma, Predictors of Cognitive Decline Among Normal Individuals (BIOCARD) and the Alzheimer's Disease Neuroimaging Initiative (ADNI) studies, S. Varma et al., "Alpha-2 macroglobulin in Alzheimer's disease: a marker of neuronal injury through the RCAN1 pathway," Molecular Psychiatry, vol. 22, no. 1, pp. 13-23, 2017.

[68] Y. Sugimura-Wakayama, W. Katagiri, M. Osugi et al., "Peripheral nerve regeneration by secretomes of stem cells from human exfoliated deciduous teeth," Stem Cells and Development, vol. 24, no. 22, pp. 2687-2699, 2015.

[69] K. Matsubara, Y. Matsushita, K. Sakai et al., "Secreted ectodomain of sialic acid-binding Ig-like lectin-9 and monocyte chemoattractant protein-1 promote recovery after rat spinal cord injury by altering macrophage polarity," The Journal of Neuroscience, vol. 35, no. 6, pp. 2452-2464, 2015.

[70] N. El-Moataz, B. Ahmed, M. Murakami, Y. Hirose, and M. Nakashima, "Therapeutic potential of dental pulp stem cell secretome for Alzheimer's disease treatment: an in vitro study," Stem Cells International, vol. 2016, Article ID 8102478, 11 pages, 2016.

[71] M. Noda and A. Suzumura, "Sweepers in the CNS: microglial migration and phagocytosis in the Alzheimer disease pathogenesis," International Journal of Alzheimer's Disease, vol. 2012, 11 pages, 2012.

[72] M. K. Kolar, V. N. Itte, P. J. Kingham, L. N. Novikov, M. Wiberg, and P. Kelk, "The neurotrophic effects of different human dental mesenchymal stem cells," Scientific Reports, vol. 7, no. 1, 2017.

[73] X. Zhuang, X. Xiang, W. Grizzle et al., "Treatment of brain inflammatory diseases by delivering exosome encapsulated anti-inflammatory drugs from the nasal region to the brain," Molecular Therapy, vol. 19, no. 10, pp. 1769-1779, 2011.

[74] Y. H. Huang, J. C. Yang, C. W. Wang, and S. Y. Lee, "Dental stem cells and tooth banking for regenerative medicine," Journal of Experimental and Clinical Medicine, vol. 2, no. 3, pp. 111-117, 2010.

[75] J. Mey, G. Brook, D. Hodde, and A. Kriebel, "Electrospun fibers as substrates for peripheral nerve regeneration," Advances in Polymer Science, vol. 246, no. 1, pp. 131-170, 2012. 


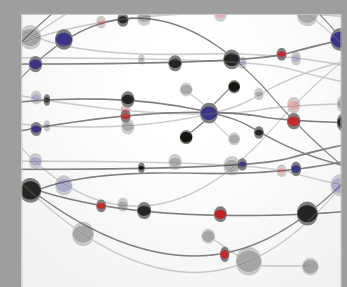

The Scientific World Journal
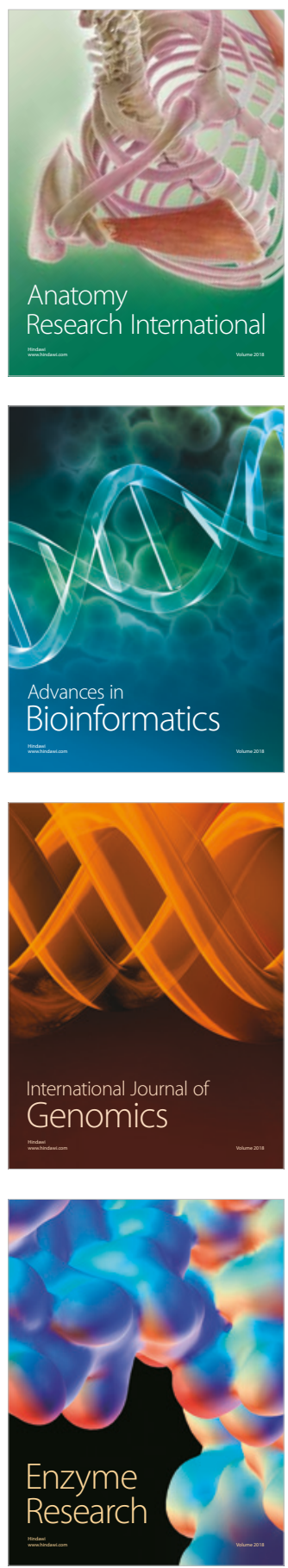
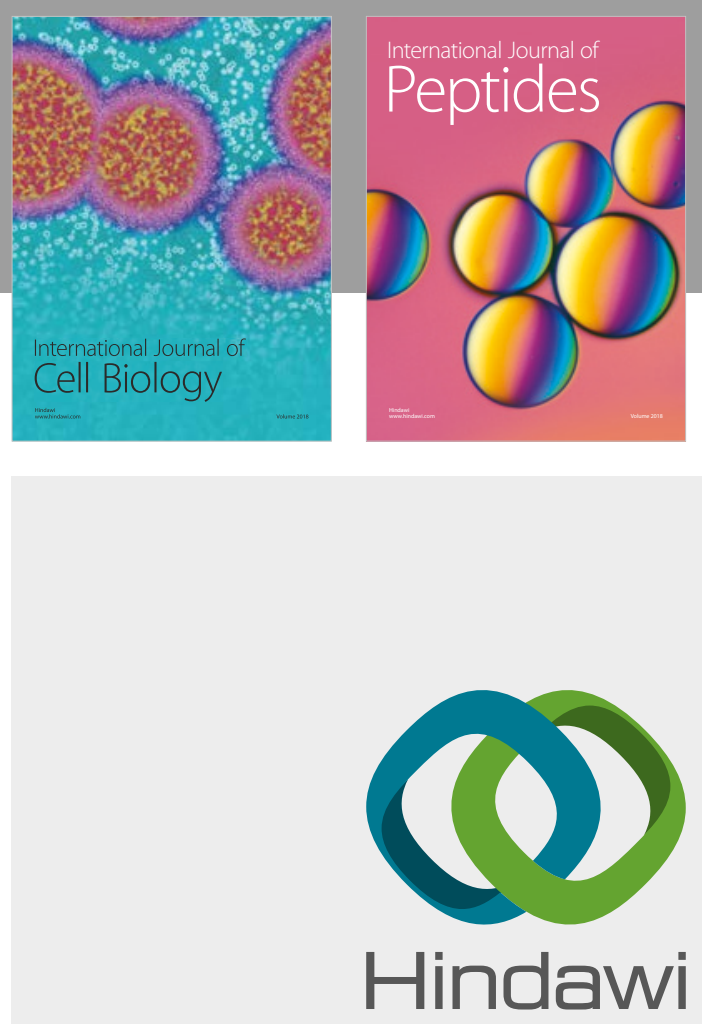

Submit your manuscripts at

www.hindawi.com
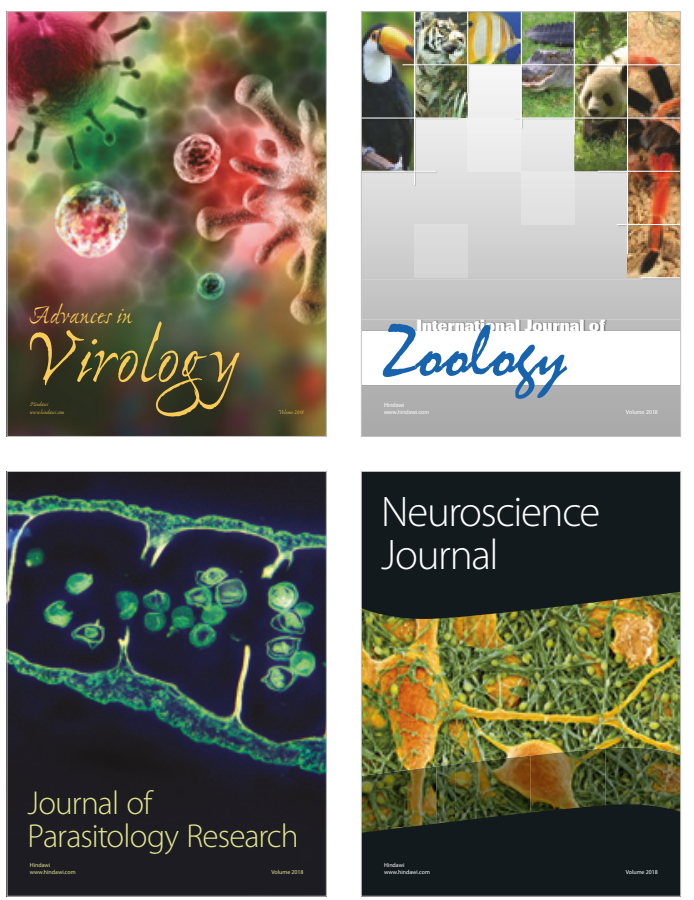
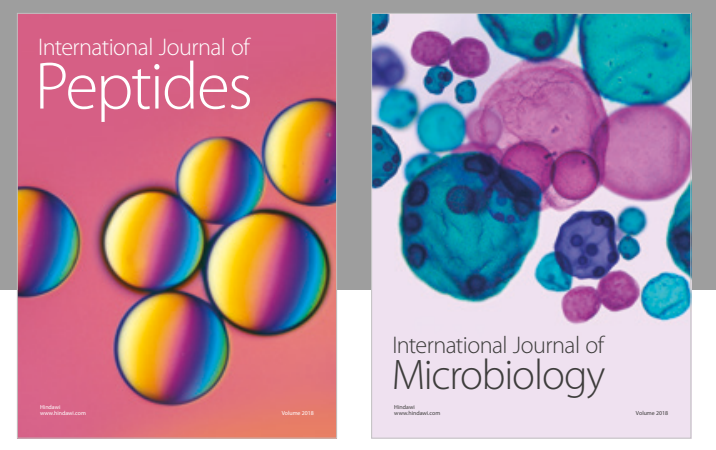

nternational Journal of Microbiology
Journal of
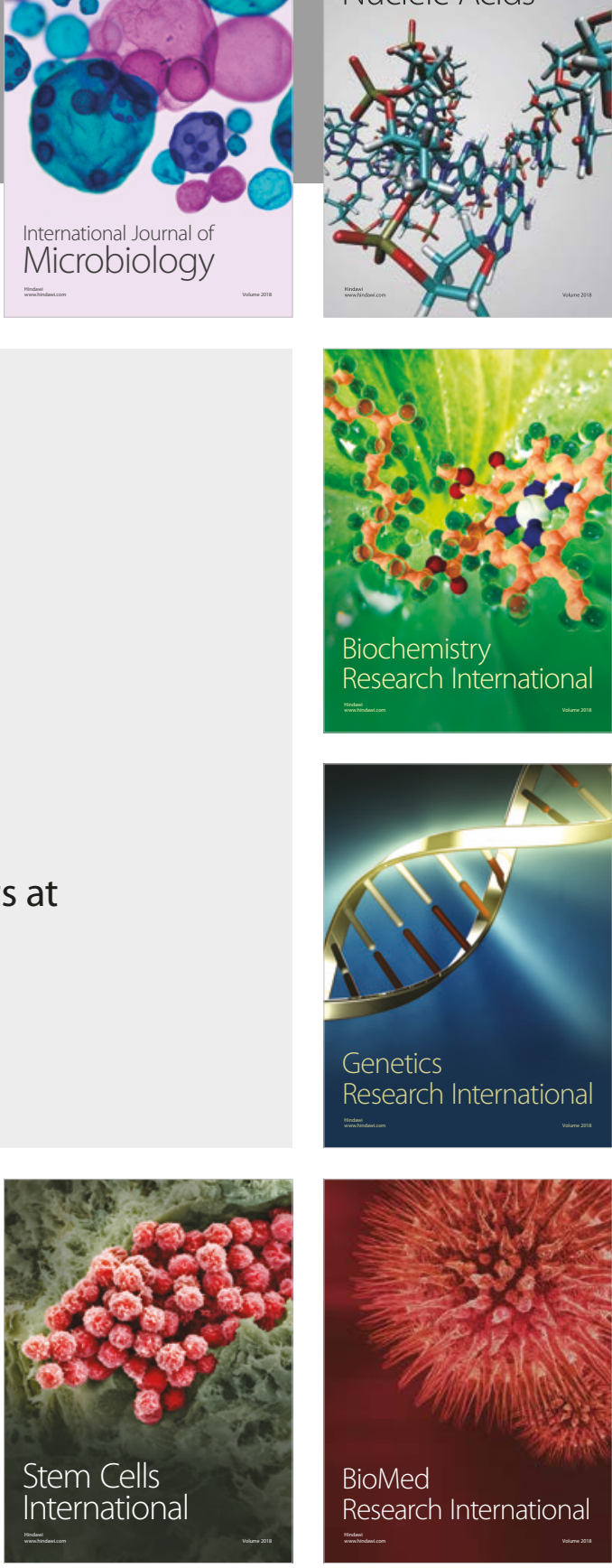
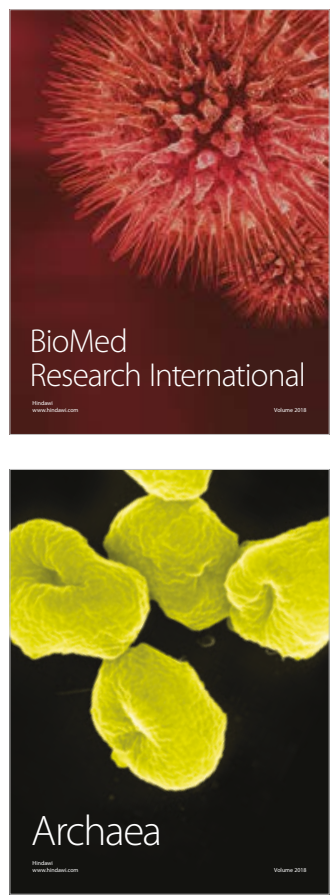